\title{
Numeri caratteristici definitivi dei flocculi di calcio per gli anni internazionali di quiete solare
}

\author{
G. Godoli - F. Mazzucconi - B. Monsignori Fossi
}

Ricevuto il 1 Febbraio 1967

\begin{abstract}
Riassdnto. - Si danno i numeri caratteristici definitivi dei flocculi di calcio per gli Anni Internazionali di Quiete Solare secondo il materiale di osservazione raccolto al World Date Center di Arcetri grazie al programma di collaborazione internazionale.

Questi numeri caratteristici sostituiscono quelli preliminari, già pubblicati nel bollettino annuale delle osservazioni eseguite all'Osservatorio Astrofisico di Arcetri (12, 13), i quali vennero stimati in base alle sole osservazioni eseguite alla torre solare di Arcetri.
\end{abstract}

Summary. - The definitive character figures of the Ca bright flocculi for the International Quiet Sun Years are given. The observational material used has been collected at Arcetri World Data Center for the Ca bright flocculi, as a part of the IQSY international programme.

These character figures take the place of those already published $\left({ }^{12,13}\right)$ according to the only observations carried out at the Arcetri solar tower.

1. - Determinazioni di numeri caratteristici giornalieri dei flocculi solari di calcio sono state pubblicate, per il periodo 1950-1960, in questa sede $\left({ }^{1 \cdot 9}\right)$ e per gli anni 1961, 1963, 1964, 1965 nel bollettino annuale delle osservazioni eseguite all'Osservatorio Astrofisico di Arcetri ( $\left.{ }^{10-13}\right)$.

Per queste determinazioni è stato utilizzato il materiale spettroeliografico raccolto alla torre solare di Arcetri nella radiazione della componente $\Pi_{2,3,2}$ della riga $\pi$ del calcio una volta ionizzato.

Dal 24 Ottobre 1961 al 9 Ottobre 1963, non sono state eseguite determinazioni di numeri caratteristici dei flocculi di calcio dato che le osservazioni alla torre solare sono state sospese per procedere alla sostituzione della cupola e dei servo-meccanismi di comando. 
2. - Come è noto, sin dall'inizio dell'Anno Geofisico Internazionale, AGI (10 Luglio 1957-31 Dicembre 1958), l'Osservatorio Astrofisico di Arcetri è Centro IIondiale (World Data Center, WDC) per la raccolta e l'elaborazione dei dati relativi ai floceuli solari in radiazione $K$ del Ca II.

Grazie a questa collaborazione internazionale, il materiale di osservazione copre tutti i giorni relativi alla campagna AGI con la sola eccezione del giorno

\section{Dicembre 1957}

e tutti quelli relativi alla campagna degli Anni Internazionali di Quiete Solare, IQSY (10 Gennaio 1961-31 Dicembre 1965) con la sola eccezione del giorno

26 Dicembre 1964.

Le copie e gli originali inviati dai vari istituti sono stati riportati a negativi su lastra rispettivamente con una o due inversioni fotografiche. Il diametro dell'immagine solare è stato sempre riportato alla misura di $63 \mathrm{~mm}$.

Dill materiale di osservazione così elaborato, è stata costituita una collezione, il più omogrenea possibile, di uno spettroeliogramma al giorno.

3. - Questa collezione era già stata utilizzata per la compilazione delle mappe dei floceuli solari in radiazione $K$ del Ca II per l'AGI $\left(^{14-16}\right)$ e gli IQSY ${ }^{(7-18)}$.

Successivamente, la collezione relativa all'AGI è stata utilizzata anche per una determinazione dei numeri caratteristici in quanto le nuove stime, eseguite su materiale che presentava soltanto una lacuna di un giorno, sarebbero state senz'altro più complete ed anche più precise di quelle già eseguite sul solo materiale di Arcetri $\left.{ }^{19}\right)$.

Col presente lavoro ci siamo proposti, nello stesso spirito, di eseguire sulla base della collezione internazionale, una nuova stima anche per il periodo degli IQSY.

Abbiamo gia notato $\left({ }^{7},{ }^{19}\right)$ che, sebbene dal $1^{\circ}$ Gennaio 1957 l'attività dei floceuli di calcio venga seguita all'Osservatorio Astrofisico di Arcetri rilevando area e posizione dei singoli fenomeni in quanto questo metodo di determinare l'attività dei flocculi è più informativo della stima del numero caratteristico e sebbene il numero caratteristico sia una funzione nota dell'area proiettata, tuttavia è opportuno continuare 
Tabella I. - Numeri caratteistici pefinitivi JeI flocculi do CAlcio Per gli Axwi Ixternazioxali di Quiete Solare. (Periodo l Gennaio 1964 - 30 Giugno 1964).

\begin{tabular}{|c|c|c|c|c|c|c|c|c|c|c|c|c|}
\hline \multirow{2}{*}{$\begin{array}{c}\text { Mese } \\
-\rightarrow\end{array}$} & \multicolumn{2}{|c|}{ Gennaio } & \multicolumn{2}{|c|}{ Febbraio } & \multicolumn{2}{|c|}{ Marzo } & \multicolumn{2}{|c|}{ Aprile } & \multicolumn{2}{|c|}{ Maggrio } & \multicolumn{2}{|c|}{ Giugno } \\
\hline & n. c. & 0 & n.e. & 0 & n.c. & 0 & n.c. & 0 & II. c. & 0 & n.c. & 0 \\
\hline 1 & 0.1 & K & 0.0 & $K$ & 1.3 & MIM & 0.6 & Ii & 0.2 & $\mathrm{MLI}$ & 0.4 & MLII \\
\hline 2 & 0.2 & $\Lambda$ & 0.0 & $\mathrm{~K}$ & 1.1 & MIMI & 0.8 & II & 0.2 & $M I M$ & 0.4 & Min \\
\hline 3 & 0.3 & $\Lambda$ & 0.0 & $A$ & 1.1 & $\mathbf{K}$ & 0.8 & $\mathrm{I}$ & 0.2 & $\mathrm{MMI}$ & 0.4 & MIM \\
\hline 4 & 0.3 & $M M$ & 0.0 & $\mathrm{~K}$ & 1.0 & $\mathrm{~K}$ & 0.9 & $M M$ & 0.3 & MLII & 0.3 & $\Lambda$ \\
\hline 5 & 0.3 & $A$ & 0.0 & $K$ & 1.0 & $\mathrm{~K}$ & 1.0 & $\mathrm{MM}$ & 0.4 & $M M$ & 0.3 & $\Lambda$ \\
\hline 6 & 0.4 & MIN & 0.0 & A & 0.9 & $\mathrm{~K}$ & 0.8 & $\mathrm{~K}$ & 0.4 & $\Lambda$ & 0.3 & $A$ \\
\hline 7 & 0.3 & $A$ & 0.0 & A & 0.7 & $\mathrm{~K}$ & 0.8 & $\mathrm{~K}$ & 0.5 & MII & 0.3 & $\Lambda$ \\
\hline 8 & 0.3 & $\Lambda$ & 0.1 & A & 0.6 & $\mathrm{~K}$ & 0.7 & $K$ & 0.5 & MM & 0.2 & $\Lambda$ \\
\hline 9 & 0.4 & A & 0.2 & A & 0.4 & $\mathrm{~K}$ & 0.6 & $\Lambda$ & 0.5 & MMI & 0.4 & MIII \\
\hline 10 & 0.5 & $\mathrm{~K}$ & 0.2 & $\Lambda$ & 0.3 & $\mathrm{~K}$ & 0.6 & $A$ & 0.6 & $M M$ & 0.5 & $\mathrm{~A}$ \\
\hline 11 & 0.5 & $\mathrm{~K}$ & 0.2 & $\mathrm{~K}$ & 0.4 & $\mathrm{~K}$ & 0.6 & MLI & 0.5 & $\operatorname{MNI}:$ & 0.6 & $\Lambda$ \\
\hline 12 & 0.7 & A & 0.0 & $A$ & 0.5 & MII & 0.7 & $\mathrm{~K}$ & 0.3 & A & 0.7 & A \\
\hline 13 & 0.7 & $\kappa$ & 0.0 & $\mathrm{~K}$ & 0.8 & $M \mathrm{II}^{2}$ & 0.6 & A & 0.3 & 1 & 0.8 & $\Lambda$ \\
\hline 14 & 0.8 & $\mathrm{~K}$ & 0.2 & MII & 0.7 & $\mathrm{~K}$ & 0.6 & $\mathrm{~K}$ & 0.3 & $" A$ & 0.9 & MNI \\
\hline 15 & 0.8 & $\mathrm{~K}$ & 0.4 & MIM & 0.7 & $\mathrm{~K}$ & 0.6 & A & 0.4 & $A^{2}$ & 0.9 & $\mathrm{~K}$ \\
\hline 16 & 0.8 & $\mathrm{~K}$ & 0.5 & $\mathrm{~K}$ & 0.7 & $\mathrm{MLI}$ & 0.5 & $A$ & 0.4 & ${ }_{A}$ & 1.0 & A \\
\hline 17 & 0.7 & A & 0.7 & $\mathrm{~K}$ & 0.6 & MIM & 0.5 & 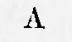 & 0.5 & $A$ & 1.0 & $\Lambda$ \\
\hline 18 & 0.6 & A & 0.9 & $\mathrm{~K}$ & 0.5 & MM & 0.4 & $K$ & 0.5 & $\Lambda$ & 1.0 & $\mathrm{MIN}$ \\
\hline 19 & 0.7 & A & 0.9 & $\mathrm{~K}$ & 0.5 & $M M$ & 0.5 & $K$ & 0.5 & I & 0.9 & $\mathrm{MIM}$ \\
\hline 20 & 0.6 & A & 1.1 & MII & 0.5 & $\mathrm{~K}$ & 0.5 & $\mathrm{~K}$ & 0.5 & K & 0.8 & MML \\
\hline 21 & 0.5 & $\Lambda$ & 1.3 & MINI & 0.5 & MLM & 0.6 & $\mathrm{~K}$ & 0.5 & $K$ & 0.7 & MLAI \\
\hline 22 & 0.7 & $\Lambda$ & 1.3 & $\mathrm{~K}$ & 0.7 & MMI & 0.6 & $\Lambda$ & 0.4 & $\Lambda$ & 0.6 & $\Lambda$ \\
\hline 23 & 0.7 & $\Lambda$ & 1.4 & $\mathrm{I}$ & 0.9 & $\mathbf{K}$ & 0.6 & $\mathrm{I}$ & 0.4 & $\mathrm{M} \mathrm{M}$ & 0.5 & $\Lambda$ \\
\hline 24 & 0.8 & A & 1.5 & $\mathrm{~K}$ & 0.9 & $\mathrm{~K}$ & 0.5 & $\mathrm{I}$ & 0.4 & MLI & 0.4 & MIM \\
\hline 25 & 0.7 & K & 1.5 & II & 0.8 & $K$ & 0.5 & $A$ & 0.4 & $\mathrm{MMI}$ & 0.2 & MM \\
\hline 26 & 0.7 & $\mathrm{~K}$ & 1.5 & $K$ & 0.8 & $\mathbf{K}$ & 0.5 & A & 0.3 & A & 0.0 & $\Lambda$ \\
\hline 27 & 0.6 & $\mathbf{K}$ & 1.5 & $K$ & 0.7 & $\mathbf{K}$ & 0.4 & A & 0.5 & $\mathrm{MMI}$ & 0.0 & MMI \\
\hline 28 & 0.6 & $\mathbf{K}$ & 1.5 & II & 0.6 & $\mathbf{K}$ & 0.3 & A & 0.4 & MLII & 0.0 & $\mathrm{MM}$ \\
\hline 29 & 0.7 & $\mathbf{K}$ & 1.5 & MIM & 0.5 & $\mathrm{~K}$ & 0.2 & $\mathrm{I}$ & 0.4 & $\Lambda$ & 0.0 & A \\
\hline 30 & 0.6 & A & - & & 0.5 & $\mathrm{~K}$ & 0.2 & $\mathrm{~K}$ & 0.5 & IIMI & 0.1 & $A$ \\
\hline 31 & 0.5 & K & - & & 0.6 & MM & - & & 0.4 & $\mathrm{MLM}$ & - & - \\
\hline $\begin{array}{c}\text { Media } \\
\text { mensile } \\
\rightarrow\end{array}$ & \multicolumn{2}{|c|}{0.55} & \multicolumn{2}{|c|}{0.63} & \multicolumn{2}{|c|}{0.70} & \multicolumn{2}{|c|}{0.58} & \multicolumn{2}{|c|}{0.41} & \multicolumn{2}{|c|}{0.49} \\
\hline
\end{tabular}


Tabella 11. - Numeri Caratteristici definitivi dei flocculi di Calcio per gei Axxi Ixtelexaztonafi di Quiete Solale. (Periodo 1 Luglio 1964 - 31 Dicembre 1964).

\begin{tabular}{|c|c|c|c|c|c|c|c|c|c|c|c|c|}
\hline \multirow{2}{*}{ 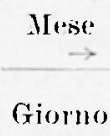 } & \multicolumn{2}{|c|}{ Lugrlio } & \multicolumn{2}{|c|}{ Agosto } & \multicolumn{2}{|c|}{ Settembre } & \multicolumn{2}{|c|}{ Ottobre } & \multicolumn{2}{|c|}{ Novembre } & \multicolumn{2}{|c|}{ Dicembre } \\
\hline & II.c. & 0 & il.c. & 0 & n.c. & 0 & n.c. & 0 & II. c. & 0 & n.c. & 0 \\
\hline l & 0.1 & A & 0.3 & $A$ & 0.3 & $A$ & 0.6 & $\Lambda$ & 0.9 & MII & 0.2 & K \\
\hline 2 & 0.2 & $\Lambda$ & 0.4 & MIMI & 0.4 & IIMI & 0.7 & MIN & 0.8 & MIM & 0.4 & MI \\
\hline 3 & 0.3 & $\Lambda$ & 0.4 & $\Lambda$ & 0.5 & $\lambda$ & 0.8 & MLI & 0.8 & $A$ & 0.4 & $\mathrm{~K}$ \\
\hline 4 & 0.4 & MII & 0.4 & $\Lambda$ & 0.5 & $A$ & 0.9 & $M[M$ & 0.8 & $\mathrm{~K}$ & 0.5 & $\Lambda$ \\
\hline 5 & 0.4 & MLMI & 0.3 & $\Lambda$ & 0.5 & MM & 0.9 & $\Lambda$ & 0.7 & $A$ & 0.7 & Ii \\
\hline 6 & 0.4 & K & 0.2 & $\lambda$ & 0.6 & MuI & 0.9 & $\Lambda$ & 0.4 & $A$ & 0.9 & $M M[$ \\
\hline 7 & 0.4 & $\mathrm{~K}$ & 0.3 & $A$ & 0.6 & $A$ & 1.0 & $\Lambda$ & 0.4 & $\mathrm{~K}$ & 0.9 & K \\
\hline 8 & 0.4 & $\mathrm{~K}$ & 0.3 & 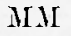 & 0.6 & $\lambda$ & 0.9 & K & 0.4 & $M M[$ & 1.0 & I \\
\hline 9 & 0.5 & $M \mu \Gamma$ & 0.3 & $A$ & 0.7 & 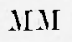 & 0.7 & $\Lambda$ & 0.4 & $\mathrm{~K}$ & 1.1 & MIM \\
\hline 10 & 0.5 & $\mathrm{MM}$ & 0.4 & MMI & 0.8 & $\Lambda$ & 0.4 & MLI & 0.5 & $M M[$ & 1.0 & $A$ \\
\hline 11 & 0.5 & MII & 0.4 & $A$ & 0.8 & MM & 0.4 & MLI & 0.5 & MM & 1. 2 & I \\
\hline 12 & 0.5 & $\mathrm{MM}$ & 0.5 & MLI & 0.7 & MIII & 0.3 & K & 0.5 & in & 1. 2 & $\mathrm{~K}$ \\
\hline 13 & 0.4 & $\Lambda$ & 0.6 & $\Lambda$ & 0.7 & $\Lambda$ & 0.4 & $\mathrm{~K}$ & 0.4 & $\Lambda$ & 1.1 & $\mathrm{~K}$ \\
\hline 14 & 0.4 & $\mathrm{MM}$ & 0.7 & $\mathrm{~K}$ & 0.6 & $M$ & 0.4 & MII & 0.4 & $\Lambda$ & 1. 1 & $\mathrm{~K}$ \\
\hline 15 & 0.3 & $\mathrm{M} M$ & 0.9 & MLI & 0.4 & $\Lambda$ & 0.5 & $\mathrm{~T}$ & 0.4 & $\mathrm{~K}$ & 1.1 & $M M \Gamma$ \\
\hline 16 & 0.3 & $\Lambda$ & 0.9 & $\Lambda$ & 0.2 & MIM & 0.5 & $\mathrm{~K}$ & 0.5 & I & 1.0 & $\mathrm{~K}$ \\
\hline 17 & 0.3 & $\mathrm{MM}$ & 0.8 & A & 0.1 & MIM & 0.5 & MII & 0.6 & MMI & 1.0 & K \\
\hline 18 & 0.4 & MII & 0.7 & $\mathrm{I}$ & 0.0 & $\Lambda$ & 0.6 & $M M$ & 0.7 & MII & 1.0 & $K$ \\
\hline 19 & 0.4 & ММ & 0.6 & $\Lambda$ & 0.1 & A & 0.6 & $\Lambda$ & 0.7 & $A$ & 1.1 & K \\
\hline 20 & 0.4 & МГМ & 0.5 & K & 0.1 & $\mathrm{MM}$ & 0.7 & $K$ & 0.6 & $M[M$ & 1. 2 & $\mathbf{I}$ \\
\hline 21 & 0.3 & ММ & 0.5 & A & 0.1 & $A$ & 0.8 & 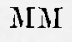 & 0.5 & $\mathrm{~T}$ & 1. 2 & MM \\
\hline 22 & 0.2 & $\Lambda$ & 0.4 & K & 0.2 & МाМ & 0.8 & K & 0.5 & MIM & 1.0 & $\Lambda$ \\
\hline 23 & 0.2 & MLМI & 0.2 & $A$ & 0.2 & A & 0.9 & $\mathrm{~K}$ & 0.4 & A & 0.9 & A \\
\hline 24 & 0.1 & $A$ & 0.2 & $K$ & 0.2 & $A$ & 0.9 & $K$ & 0.5 & $M \Pi[$ & 0.9 & $F$ \\
\hline 25 & 0.2 & $M M$ & 0.3 & $\Lambda$ & 0.1 & $\Lambda$ & 0.8 & $\mathrm{~K}$ & 0.5 & $\mathrm{MM}$ & 1.0 & $\mathbf{T}$ \\
\hline 26 & 0.2 & $\Lambda$ & 0.3 & $\Lambda$ & 0.1 & $A$ & 0.9 & MII & 0.4 & $M \Pi$ & - & - \\
\hline 27 & 0.1 & $A$ & 0.3 & $A$ & 0.1 & $\Lambda$ & 0.9 & $\mathrm{~K}$ & 0.4 & $\mathrm{I}$ & 1.0 & MМL \\
\hline 28 & 0.0 & MM & 0.2 & $A$ & 0.2 & MMI & 1.0 & Ii & 0.3 & $\mathrm{~K}$ & 0.8 & I \\
\hline 29 & 0.0 & MM & 0.2 & $A$ & 0.3 & A & 1.0 & $A$ & 0.3 & K & 0.7 & $K$ \\
\hline 30 & 0.0 & MM & 0.2 & $\mathrm{MM}$ & 0.5 & $\Lambda$ & 1.0 & $A$ & 0.4 & $\mathrm{~K}$ & 0.6 & K \\
\hline 31 & 0.2 & $A$ & 0.2 & $\Lambda$ & $-\cdots$ & - & 1.0 & MIII & - & - & 0.6 & Ii \\
\hline $\begin{array}{l}\text { Modia } \\
\text { mensile }\end{array}$ & \multicolumn{2}{|c|}{0.29} & \multicolumn{2}{|c|}{0.42} & \multicolumn{2}{|c|}{0.37} & \multicolumn{2}{|c|}{0.73} & \multicolumn{2}{|c|}{0.52} & \multicolumn{2}{|c|}{0.90} \\
\hline \multicolumn{13}{|c|}{$\begin{array}{l}\text { Media semestrale } 0,54 \\
\text { Media annuale } 0.55\end{array}$} \\
\hline
\end{tabular}


Tabella 11I. - Numeri Caratteristici deFinitivi dei llocculi di calcio Per gli Anni Internazionali di Quiete Solare. (Periodo l Gemnaio 1965 - 30 Giugno 1965).

\begin{tabular}{|c|c|c|c|c|c|c|c|c|c|c|c|c|}
\hline \multirow{2}{*}{$\begin{array}{r}\stackrel{\text { Mese }}{\rightarrow} \\
\text { Giorno }\end{array}$} & \multicolumn{2}{|c|}{ Gennaio } & \multicolumn{2}{|c|}{ Febbraio } & \multicolumn{2}{|c|}{ Marzo } & \multicolumn{2}{|c|}{ Aprile } & \multicolumn{2}{|c|}{ Maggio } & \multicolumn{2}{|c|}{ Giugno } \\
\hline & n.c. & 0 & n.c. & o & n.c. & 0 & n.c. & o & n.c. & 0 & n.c. & 0 \\
\hline 1 & 0.8 & $\mathbf{K}$ & 1. 2 & $\mathrm{~K}$ & 1.1 & $M M$ & 0.6 & $\mathrm{IJ}$ & 0.2 & MNI & 0.8 & $M M$ \\
\hline 2 & 0.9 & $\mathrm{~K}$ & 1.4 & K & 1.0 & MLMI & 0.6 & MIM & 0.3 & MIM & 0.9 & $\mathrm{~K}$ \\
\hline 3 & 1.2 & MIM & 1.2 & $A$ & 1.0 & $\mathrm{MI}$ & 0.4 & MM & 0.4 & MIM & 1.0 & $\mathrm{~K}$ \\
\hline 4 & 1.4 & $\mathbf{K}$ & 1.0 & A & 1.0 & $\mathrm{~T}$ & 0.2 & MMI & 0.5 & $M$ & 1.1 & $\mathrm{~K}$ \\
\hline 5 & 1.3 & $\mathbf{K}$ & 1.0 & $\mathrm{~K}$ & 1.1 & $\mathrm{~T}$ & 0.0 & MIM & 0.5 & MМI & 1.1 & $\mathrm{~K}$ \\
\hline 6 & 1.0 & $A$ & 0.8 & $\mathrm{~K}$ & 1.0 & $M$ & 0.1 & MLI & 0.6 & MMI & 1.0 & $\mathrm{~K}$ \\
\hline 7 & 0.9 & $\Lambda$ & 0.8 & i & 0.9 & $\mathrm{~T}$ & 0.3 & MLM & 0.7 & MML & 1.2 & MM \\
\hline 8 & 0.9 & $\mathbf{K}$ & 1.0 & $\mathrm{~K}$ & 0.6 & $\mathbf{K}$ & 0.5 & $\mathrm{~K}$ & 0.8 & MNI & 1. 3 & ММ \\
\hline 9 & 0.9 & $\mathbf{K}$ & 1.0 & $A$ & 0.3 & $\mathrm{~L}$ & 0.6 & $\mathrm{~K}$ & 0.8 & MII & 1. 2 & $M$ \\
\hline 10 & 1.0 & $\mathbf{K}$ & 0.6 & $A$ & 0.4 & 'T' & 0.7 & Ii & 0.7 & $\mathrm{MNI}$ & 1.0 & МГМ \\
\hline 11 & 1.2 & $\mathbf{K}$ & 0.6 & $A$ & 0.4 & MМ & 0.8 & MIM & 0.8 & ММ & 1.0 & $\Lambda$ \\
\hline 12 & 1.0 & $\mathrm{~K}$ & 0.5 & A & 0.4 & MMI & 1.0 & $M M$ & 0.9 & $M M$ & 1.2 & $M M$ \\
\hline 13 & 0.8 & $\mathbf{K}$ & 0.6 & A & 0.6 & $M M$ & 1.0 & $\mathrm{M} M$ & 0.9 & MLI & 1.2 & МूM \\
\hline 14 & 0.7 & $\mathbf{K}$ & 0.6 & $\mathrm{MIM}$ & 0.5 & $\mathrm{~T}$ & 1.2 & $M L I$ & 0.9 & $M$ & 1.3 & A \\
\hline 15 & 0.5 & $\mathrm{~K}$ & 0.6 & $M M$ & 0.6 & MIMI & 1.2 & $\mathrm{I}$ & 1.0 & MMI & 1. 3 & A \\
\hline 16 & 0.1 & $\mathrm{~K}$ & 0.5 & $A$ & 0.6 & $M \Pi \Gamma$ & 1.3 & K & 1.2 & MMI & 1.3 & $\mathrm{k}$ \\
\hline 17 & 0.5 & $\mathrm{~K}$ & 0.4 & A & 0.6 & L & 1.3 & $\mathrm{~K}$ & 1.3 & $\mathbf{K}$ & 1.4 & $M M$ \\
\hline 18 & 0.7 & $\mathrm{~K}$ & 0.4 & II & 0.7 & $M M$ & 1.2 & MIMI & 1.4 & $\mathbf{K}$ & 1.4 & A \\
\hline 19 & 0.7 & $M$ & 0.3 & I & 0.7 & ММГ & 1.1 & MLM & 1.5 & $\mathbf{K}$ & 1.4 & A \\
\hline 20 & 0.7 & $A$ & 0.4 & $\mathrm{~K}$ & 0.7 & $\mathrm{MMI}$ & 1.0 & MIM & 1.5 & $\mathbf{K}$ & 1.3 & MMI \\
\hline 21 & 0.9 & $\mathrm{~K}$ & 0.4 & $\mathrm{~K}$ & 0.6 & MMI & 1.0 & МलМ & 1.6 & $\mathbf{K}$ & 1.2 & A \\
\hline 22 & 0.9 & $\mathrm{~K}$ & 0.4 & $\mathrm{~K}$ & 0.6 & $\mathrm{~K}$ & 0.9 & MLM & 1.5 & $\mathbf{K}$ & 1.0 & A \\
\hline 23 & 0.8 & $\mathrm{k}$ & 0.5 & ММ & 0.3 & $\mathrm{~K}$ & 0.8 & MLI & 1.4 & $\mathrm{~K}$ & 0.8 & A \\
\hline 24 & 0.8 & $\mathrm{~K}$ & 0.6 & $\mathrm{~K}$ & 0.2 & K & 0.6 & MMI & 1.4 & A & 0.8 & $\Lambda$ \\
\hline 25 & 0.9 & $\mathrm{~K}$ & 0.8 & $\mathrm{~K}$ & 0.4 & $\mathrm{~K}$ & 0.5 & 'T & 1.4 & $\boldsymbol{\Lambda}$ & 0.9 & $\mathrm{~A}$ \\
\hline 26 & 0.9 & $\mathrm{~K}$ & 1.0 & MIMI & 0.6 & MI & 0.7 & K & 1.4 & $A$ & 1.1 & MMI \\
\hline 27 & 0.9 & K & 1.1 & MMI & 0.8 & $\mathrm{MM}$ & 0.6 & 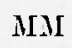 & 1.2 & MIM & 1.1 & MM \\
\hline 28 & 1.0 & $\mathrm{~K}$ & 1.2 & MM & 0.8 & $M$ & 0.4 & MIM & 1.0 & $\mathbf{K}$ & 1.1 & MMI \\
\hline 29 & 1.2 & $\mathbf{K}$ & - & - & 0.7 & $\mathrm{~K}$ & 0.4 & MLM & 0.9 & $\mathbf{K}$ & 1.1 & MMI \\
\hline 30 & 1.3 & $\mathrm{~K}$ & - & - & 0.6 & $\mathbf{K}$ & 0.2 & $\mathrm{~K}$ & 0.8 & $M M$ & 1.0 & A \\
\hline 31 & 1.3 & $\mathbf{K}$ & - & - & 0.6 & MLMI & - & - & 0.8 & MII & $\cdot-$ & - \\
\hline$\underset{\rightarrow}{\substack{\text { Media } \\
\text { mousile } \\
\rightarrow}}$ & \multicolumn{2}{|c|}{0.91} & \multicolumn{2}{|c|}{0.75} & \multicolumn{2}{|c|}{0.66} & \multicolumn{2}{|c|}{0.71} & \multicolumn{2}{|c|}{0.98} & \multicolumn{2}{|c|}{1.12} \\
\hline \multicolumn{13}{|c|}{ Media semestrale } \\
\hline
\end{tabular}


Tabela IV. - Numeri Caratteristici Definitivi deI flocculi di Calcio per gli AnNi Internazionali d) Quiete Solare. (Periodo 1 Iugglio 1965 - 31 Dicembre 1965).

\begin{tabular}{|c|c|c|c|c|c|c|c|c|c|c|c|c|}
\hline \multirow{2}{*}{$\begin{array}{l}\stackrel{\text { Mese }}{\rightarrow} \\
\text { Giorno }\end{array}$} & \multicolumn{2}{|c|}{ Luglio } & \multicolumn{2}{|c|}{ Agosto } & \multicolumn{2}{|c|}{ Scttembre } & \multicolumn{2}{|c|}{ Ottobre } & \multicolumn{2}{|c|}{ Novembre } & \multicolumn{2}{|c|}{ Dicembre } \\
\hline & n.c. & 0 & n.e. & 0 & n.c. & 0 & n.c. & 0 & n.c. & 0 & n.c. & 0 \\
\hline 1 & 1.1 & $A$ & 0.6 & MIII & 0.6 & $M M I$ & 1.3 & $\Lambda$ & 1. 2 & IIUI & 0.7 & A \\
\hline 2 & 1.2 & $\Lambda$ & 0.6 & M & 0.7 & K & 1.4 & MIM & 1.2 & $\mathrm{MMI}$ & 0.7 & $\mathrm{~A}$ \\
\hline 3 & 1.2 & MIII & 0.7 & $M M$ & 0.7 & $\mathrm{~K}$ & 1.6 & MLI & 1.3 & MM & 0.7 & $\mathrm{~T}$ \\
\hline 4 & 1.2 & $\Lambda$ & 0.8 & $d$ & 0.7 & $\mathrm{~K}$ & 1.8 & $A$ & 1.2 & MII & 0.8 & $\mathrm{R}$ \\
\hline 5 & 1.1 & $\mathrm{~K}$ & 0.9 & $A$ & 0.7 & $\mathrm{~K}$ & 1.9 & $A$ & 1.0 & MHI & 0.7 & $M M$ \\
\hline 6 & 1.0 & $\Lambda$ & 1.0 & $\Lambda$ & 0.6 & K & 1.7 & $A$ & 0.9 & MLII & 0.8 & $\mathrm{~T}$ \\
\hline 7 & 0.9 & $\Lambda$ & 1.1 & $A$ & 0.7 & $\mathrm{~K}$ & 1.3 & $\Lambda$ & 1.0 & $M M$ & 0.8 & $M M$ \\
\hline 8 & 1.0 & $M M I$ & 1.1 & $A$ & 0.8 & I & 0.8 & $A$ & 1.0 & $\Lambda$ & 0.8 & $\mathrm{MM}$ \\
\hline 9 & 1.0 & MMI & 1.2 & $A$ & 0.8 & $\mathrm{~K}$ & 0.6 & A & 1.1 & MM & 0.9 & $M M$ \\
\hline 10 & 1.1 & MIM & 1.2 & $A$ & 0.8 & $A$ & 0.5 & $A$ & 1.1 & MII & 0.9 & MM \\
\hline 11 & 1.1 & MMI & 1.2 & $A$ & 0.9 & $\mathrm{M} M$ & 0.5 & $\Lambda$ & 1.1 & MII & 0.8 & K \\
\hline 12 & 1.2 & $\Lambda$ & 1.2 & $A$ & 0.9 & ММ & 0.5 & $\Lambda$ & 1.1 & $\mathrm{~T}$ & 0.7 & $\mathrm{~K}$ \\
\hline 13 & 1.2 & $\Lambda$ & 1.2 & $\Lambda$ & 0.8 & $A$ & 0.5 & $\Lambda$ & 0.9 & $\mathrm{MM}$ & 0.6 & $\mathrm{~T}$ \\
\hline 14 & 1.2 & $\mathrm{~A}$ & 1.3 & MMI & 0.7 & $\mathbf{F}$ & 0.5 & $A$ & 0.9 & $M M$ & 0.6 & $\mathrm{R}$ \\
\hline 15 & 1.1 & MM & 1.0 & $M M$ & 0.6 & $\Lambda$ & 0.5 & $\Lambda$ & 0.8 & $\mathrm{~T}$ & 0.5 & $\Lambda$ \\
\hline 16 & 0.9 & $M M$ & 0.7 & MIMI & 0.6 & $A$ & 0.5 & $\mathrm{~T}$ & 0.6 & $A$ & 0.6 & $\mathbf{T}$ \\
\hline 17 & 0.9 & MHI & 0.4 & $A$ & 0.6 & $A$ & 0.5 & $A$ & 0.5 & MIII & 0.7 & $M M$ \\
\hline 18 & 0.8 & $\Lambda$ & 0.4 & $\mathrm{MM}$ & 0.6 & $M M$ & 0.5 & MMI & 0.4 & $\mathrm{MM}$ & 0.7 & $M M$ \\
\hline 19 & 0.8 & $A$ & 0.5 & $\Lambda$ & 0.7 & $\mathrm{MMI}$ & 0.6 & MNI & 0.3 & MIM & 0.7 & MII \\
\hline 20 & 0.5 & $\mathrm{MMI}$ & 0.6 & $\Lambda$ & 0.7 & $\Lambda$ & 0.6 & $\Lambda$ & 0.4 & $M M$ & 0.5 & $\mathbf{K}$ \\
\hline 21 & 0.3 & $\mathrm{MLAL}$ & 0.7 & $\mathrm{MM}$ & 0.7 & $\mathrm{MM}$ & 0.7 & $\Lambda$ & 0.5 & $M M$ & 0.4 & $\mathrm{MM}$ \\
\hline 22 & 0.1 & $\Lambda$ & 0.7 & $M M$ & 0.5 & $A$ & 0.7 & $\Lambda$ & 0.6 & MLM & 0.3 & $\mathrm{MN}$ \\
\hline 23 & 0.1 & $\Lambda$ & 0.7 & MLI & 0.5 & $\Lambda$ & 0.8 & $\Lambda$ & 0.6 & K & 0.3 & $W$ \\
\hline 24 & 0.1 & $\Lambda$ & 0.7 & MМL & 0.4 & $\mathrm{~K}$ & 0.9 & $\Lambda$ & 0.6 & MII & 0.2 & $\mathrm{~T}$ \\
\hline 25 & 0.3 & 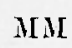 & 0.8 & $M M[$ & 0.4 & $\Lambda$ & 1.0 & $A$ & 0.6 & $\mathrm{~L}$ & 0.2 & $\mathrm{~T}$ \\
\hline 26 & 0.3 & $\mathrm{MMI}$ & 0.8 & $A$ & 0.5 & $\mathrm{~K}$ & 1.1 & $A$ & 0.6 & 'I' & 0.4 & MII \\
\hline 27 & 0.2 & $A$ & 0.8 & A & 0.7 & $\mathbf{K}$ & 1.1 & MIM & 0.6 & $\mathrm{M} M \mathrm{I}$ & 0.6 & $\mathrm{~T}$ \\
\hline 28 & 0.2 & $M M$ & 0.8 & $A$ & 1.0 & $M M$ & 1.1 & IIIM & 0.6 & MI & 0.7 & $A$ \\
\hline 29 & 0.3 & MM & 0.7 & $A$ & 1.1 & $\mathrm{~K}$ & 1.0 & MIMI & 0.7 & $\mathrm{MLI}$ & 0.8 & $A$ \\
\hline 30 & 0.4 & 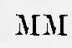 & 0.7 & $\mathrm{~K}$ & 1.1 & $\Lambda$ & 1.1 & MMI & 0.7 & ММ & 0.8 & $A$ \\
\hline 31 & 0.5 & MIM & 0.6 & $\mathrm{~K}$ & - & - & 1.1 & MMI & - & - & 0.7 & $\Lambda$ \\
\hline \multirow[t]{2}{*}{$\begin{array}{l}\text { Mredia } \\
\text { mensile }\end{array}$} & \multicolumn{2}{|c|}{0.75} & \multicolumn{2}{|c|}{0.83} & \multicolumn{2}{|c|}{0.70} & \multicolumn{2}{|c|}{0.93} & \multicolumn{2}{|c|}{0.80} & \multicolumn{2}{|c|}{0.63} \\
\hline & \multicolumn{6}{|c|}{$\begin{array}{l}\text { Media semestral } \\
\text { Media annuale }\end{array}$} & \multicolumn{2}{|c|}{$\begin{array}{l}0.77 \\
0.82\end{array}$} & & & & \\
\hline
\end{tabular}


a stimare i numeri caratteristici dei flocculi di calcio dato che vi sono elementi per ritenere che, durante fasi peculiari del ciclo solare, i due diversi metodi diano informazioni di significato diverso $\left({ }^{20}\right)$.

4. - Le stime dei numeri caratteristici sono state sempre eseguite all'Osservatorio Astrofisico di Arcetri attenendosi ai modelli distribuiti dall'Osservatorio di NLount Wilson. I modelli definiscono i punti 0, 1, 2, 3, 4, 5 della scala dei numeri caratteristici. Ad Areetri è sempre stato considerato conveniente stimare anche il decimo del numero caratteristico, mentre altri Osservatori preferiscono dare solo i cinque decimi.

I modelli originali di Mount Wilson sono costituiti da positive su carta del diametro di $100 \mathrm{~mm}$. Recentemente ad Areetri, per migliorare lá qualità della stima, si è derivata dai modelli originali di Mount Wilson una serie di modelli costituita da negative su lastra del diametro di $63 \mathrm{~mm}$. Dal 1962 i numeri caratteristici sono sempre stati stimati con l'aiuto di questi modelli.

5. - La nuova stima sistematica di numeri caratteristici è stata fatta indipendentemente da due osservatori (F.MI. e B.AL.F.); un terzo osservatore (G.G.) ha saltuariamente controllato le stime. Quando lo scarto medio delle singole determinazioni non superava 0.1 unità, veniva fatta una media delle valutazioni dei due osservatori; nei pochi casi in cui veniva riscontrato uno searto medio di magrorior entità lo spettroeliogramma veniva riesaminato.

Nelle Tabelle I-VI sono riportate le nuove stime che, dato il loro carattere di completezza, costituiramno, d'ora in poi, la serie definitiva per gli Anni Internazionali di Quiete Solare.

Nelle Tabelle è indicata anche la fonte dello spettroeliogramma analizzato secondo il codice:

$$
\begin{aligned}
& \mathrm{A}=\text { Areetri } \\
& \mathrm{F}=\text { Freiburg } \\
& \mathrm{K}=\text { Kodaikanal } \\
& \mathrm{L}=\text { Locamo } \\
& \mathrm{MIN}=\text { Me Math-Hulbert } \\
& \mathrm{M}=\text { Meudon } \\
& \mathrm{R}=\text { Roma } \\
& \mathrm{T}=\text { Tokio } \\
& \mathrm{W}=\text { Wendelstein. }
\end{aligned}
$$


Notiamo che lo scarto medio fra le nuove stime e quelle già pubblicate rientra generalmente nel limite accettato non superando il valore 0.1. Nella seguente Tabella sono riportati i giomi per cui lo scarto medio supera il valore 0.1 .

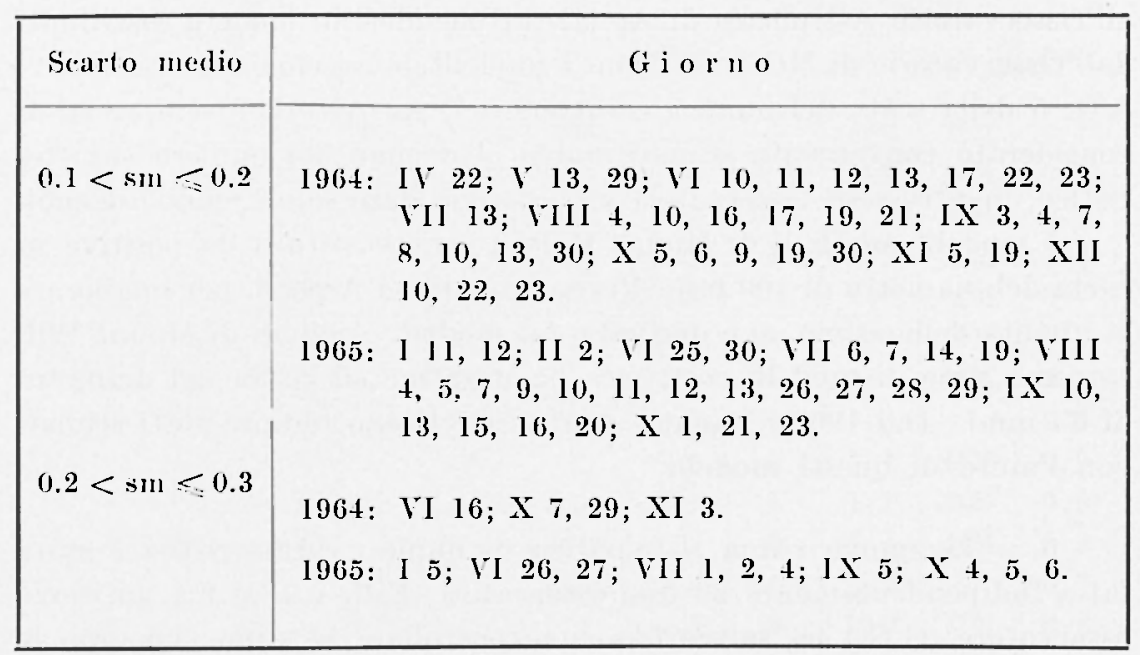

Notiamo che, in gencrale, le nuove stime tendono ad essere più elevate di quelle eseguite utilizzando il solo materiale spettroeliografico raccolto alla torre solare di Arcetri. Questa differenza sistematica, che si mantiene entro gli scarti medi accettati, è probabilmente da attribuirsi al maggior contrasto rispetto agli spettroeliogrammi di Arcetri, delle lastre della collezione internazionale, dovuto ai procedimenti fotografici. Per l'AGI questa differenza non si è riscontrata dato che, in periodi di massima dell'attività solare lo scarto medio assoluto risulta superiore, pur mantenendosi costante lo searto medio relativo.

\section{BIBLIOGRAFIA}

(1) Gonom G., Numeri caralteristici dei flocculi d'idrogeno e di calcio e dei filamenti d'idrogeno per gli anni 19.50-.j1-.5.2, "Ann. Geof. , 6, 199 (1953).

(-) Idem per lanno 1953, l.c., 7, 135 (1954).

( $\left.{ }^{3}\right)$ Idem per l'anno 1954, I.c., 8, 325 (1955).

(4) Idem per l'anno 1955, 1.c., 9, 299 (1956).

(5) Idem per l'amo 1956, l.c., 10, 133 (1957). (In collaborazione con M. P. STroccir). 
$\left(^{6}\right)$ Idem per l'anno 1957 , l.c., 10, 235 (1957).

(7) Idem per l'anno 1958, l.c., 12, 197 (1959).

(8) Idem per l'anno 1959, l.c., 13, 231 (1960).

$\left({ }^{9}\right)$ Idem per l'anno 1960, l.c., 14, 319 (1961).

$\left.{ }^{10}\right)$ Osservazioni eseguite allOsservatorio Astrofisico di Arcetri durante lanno 1961, "Oss. e Mem. dell'Oss. Astrof. di Arcetri ", 77 (1962).

(II) Ilem per l'anno 1963, l.c., 79 (1964).

(12) Idem per l'anno 1964, l.c., 80 (1965).

(1:3) Idem per l'anno 1965, 1.c., 84 (1966).

(14) Govoli G., Mappe dei Rocculi solari in radiazione K del Ca II per l'Anuo Geofisico Internazionale, I. Semestre 1 Luglio 19j\% - 31 Dicembre 19:j\%, "Oss. e Mem. dell'Oss. Astrof. di Arcetri ", 73 (1961).

(15) Idem per il Semestre 1 Gennaio 1958 - 30 Giugno 1958, 1.c., 75 (1961).

(16) Idem per il Semestre l Luglio 1958 - 31 Dicembre 1958, 1.c., 76 (1962).

(12) Govoli G., Mappe dei floceuli solari in radiazione $K$ del Ca II per gli Anni Internazionali di Quiete Solare, I. Anno 196.t, "Oss. e Mem. dell'Oss. Astrof. di Arcetri ", 82 (1966).

(18) Idem per l'anno 1965, l.c., 87 (1967).

$\left({ }^{19}\right)$ Godoli G., Mazzucconi F., Monsignori Fossi B., Numeri caratleristici definitivi dei flocculi di calcio per l'A nno Geofisico Internazionale, "Ann. Geof. ", 19, 95 (1966).

${ }^{\left({ }^{20}\right)}$ Govoli G., Altivitì dei floceuli d'idrogeno e di calcio e dei filumenti di idrogeno per il periodo 1 Luglio 1957 - 30 Giugno 1958, "Boll. di Geod. e Scienze Affini ", 17, 487 (1958). 